http://www.jfas.info

\title{
A FUZZY ART NEURAL NETWORK BASED COLOR IMAGE PROCESSING AND RECOGNITION SCHEME
}

\author{
Rashad J. Rasras
}

Albalqa' Applied University, Faculty of Engineering Technology, Amman, Jordan

Published online: 15 February 2017

\begin{abstract}
This paper is concerned with the application of an enhanced Fuzzy ART neural network algorithm for color image processing and recognition. A new category choice function was proposed that allows modifications to the category's weight vector when elements of the weight vector are smaller than elements of the input learning vector. To improve the learning process from the input data, a new learning rule was suggested. In this paper, a new method is proposed to deal with the RGB color image pixels, which enables a Fuzzy ART neural network to process the RGB color images. The application of the algorithm was implemented and tested on a set of RGB color face images
\end{abstract}

Keywords: Color image processing, RGB, Fuzzy ART

\section{INTRODUCTION}

A fuzzy ART neural network is a fuzzy version of ART-1 [1], which was developed to improve the ability of clustering. Fuzzy ART neural networks contain the basic features of ART neural network families. Carpenter et al. [1], proposed the first Fuzzy ART network, which showed the stable learning capability of recognition categories. The architecture of the fuzzy ART neural network is shown in fig. 1.

Author Correspondence, e-mail: rashad.rasras@ bau.edu.jo doi: http://dx.doi.org/10.4314/jfas.v9i1s.688 


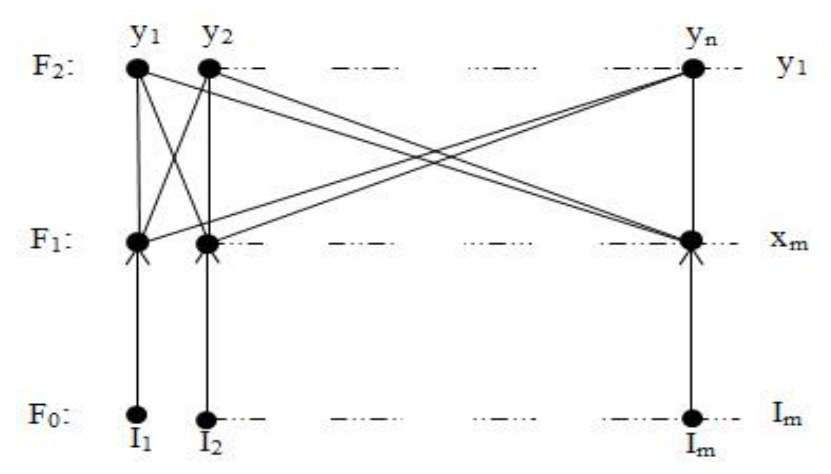

Fig. 1. Architecture of a fuzzy ART neural

The architecture of a fuzzy ART system consists of 3 layers, the first layer is the F0 layer consisting of neurons that represent a current input vector $\mathrm{I}=\left(\mathrm{I}_{1}, \ldots, \mathrm{I}_{\mathrm{m}}\right)$, followed by the $\mathrm{F} 1$ layer of $\mathrm{x}$ neurons $\mathrm{x}=\left(\mathrm{x}_{1}, \ldots, \mathrm{x}_{\mathrm{m}}\right)$ that receives both bottom-up input from $\mathrm{F} 0$ and top-down input from layer F2. The F2 layer represents the active neurons, denoted category $y$ $=\left(\mathrm{y}_{1}, \ldots, \mathrm{y}_{\mathrm{n}}\right)$. Association with each $\mathrm{F} 2$ category node $\mathrm{j}(\mathrm{j}=1, \ldots \mathrm{N})$ represents a weight vector, $\mathrm{W}_{\mathrm{j}}$ $=\left(\mathrm{w}_{\mathrm{j} 1}, \ldots, \mathrm{w}_{\mathrm{jm}}\right)$. The Fuzzy ART dynamics are determined by a choice parameter $\alpha>0, \mathrm{a}$ learning rate parameter $\beta \in[0,1]$, and a vigilance parameter $\rho \in[0,1]$. The learning and categorization algorithm of a fuzzy ART network is as follows:

Step 1: Setup weight vector.

Each category $j$ corresponds to a vector $W_{j}=W_{j 1}, \ldots, W_{j M}$ of adaptive weights, The number of potential categories $N(j=1, \ldots, N)$ is arbitrary. Initially $W_{j 1}=\ldots=W_{j M}=1$

and each category is said to be uncommitted. After a category is selected to code, it becomes committed.

Step 2: Choose a wining category.

For each input $I$ and category $j$, the choice function $T_{j}$ is defined by

$$
\mathrm{T}_{\mathrm{j}}(\mathrm{I})=\frac{\|I \wedge W j\|}{\alpha+\|W j\|}
$$

The category choice is indexed by $j$, where

$$
T_{j}=\max \left\{T_{j}, j=1, \ldots, N\right\}
$$

If more than one $T_{j}$ is maximal, the category $j$ with the smallest index is chosen.

Step 3: Resonance or reset 
Resonance occurs if the match function of the chosen category meets the vigilance criterion; that is, if

$$
\frac{\|I \wedge W j\|}{\alpha+\|W j\|} \geq \rho
$$

then learning is performed in Step 4.

Mismatch reset occurs if

$$
\frac{\|I \wedge W j\|}{\alpha+\|W j\|}<\rho
$$

Then the value of the choice function $T_{j}$ is reset to -1 for the duration of the input presentation. A new index $j$ is chosen, by Equation (2). The search process continues until the chosen $j$ satisfies Equation (3) or actives a new category.

Step 4: Perform learning process.

The weight vector of $j$ th category, $W_{j}$ is updated according to the following equation:

$$
W_{j}^{\text {new }}=\beta\left(1 \wedge W_{j}^{\text {old }}\right)+(1-\beta) W_{j}^{\text {old }}
$$

Step 5: Active a new category.

For each input $I$, if no existing category satisfies Equation (3) then a new category $j$ becomes active. Then, $W_{j}^{\text {new }}=I$.

Analysis of related literature shows that ART network families are most popular for image segmentation and recognition techniques in many applications that include face recognition in $[2,3,4]$, classification of multivariate chemical data [5], quality control of manufacturing processes [6], and classification of wireless sensor networks with missing data [7]. Models of Fuzzy ART and ART networks have developed to improve the ability of clustering. The authors in [8] have designed a network based on Fuzzy ART and proposed a new model, which has been experimentally proven to perform well in the RGB color space, and is believed to be more coherent than Fuzzy ART. In [9], Kenaya and Cheok proposed a Euclidean ART network that employed the Euclidean distance to measure the similarity and the mean of patterns for the weights of categories. In [10], the authors proposed an improved learning rule for fuzzy ART to improve learning from data. In the proposed rule [10], the 
weights of the winning category are decreased to adapt to each input.

It is known that Fuzzy ART neural networks deal with analog data. In contrast, this work develops methods to enable the fuzzy ART neural network to process color face images. As reported in [11], the recognition performance with color images produces improved results as compared with grayscale images. Color cues that are specific to an individual person may allow us to identify the person and play a role in face recognition particularly when shape cues are degraded. In this work, an enhanced algorithm was applied on RGB color images for face recognition. The algorithm in this paper can deal directly with RGB color face image pixels. The developed system is capable of recognizing color face images that are stored in memory and can otherwise store new face images that are not stored previously by adding them to the new category.

\section{ENHANCED FUZZY ART ALGORITHM}

Many studies that discuss models of fuzzy ART and their clustering ability [12, 13, 14, 15], show that learning from data is ineffective when elements of a category's weight vector are smaller than the elements of the input vector. The known learning process of a new input vector is adapted when a fuzzy subset category choice exists, and is selected over the other choices in accordance with equation 2 to updates the category's weight vector. No updating of the weights occur for category $J$ when elements of category's weight vector are smaller than elements of the input vector (since $\left(I^{\wedge} \mathrm{w}_{\mathrm{j}}=\mathrm{w}_{\mathrm{j}}\right.$.). In this work, a new equation (6) was suggested for the category choice function to replace equation 1 , as follows:

For each input I, and category $j$, a choice function $T_{j}$ is defined by:

$$
T_{J}=1-\frac{\sum_{i=1}^{n}\left|w_{J i}-I\right|}{\left\|w_{J}\right\|+\|I\|}
$$

Where $T_{j}=\max \left\{T_{j}, j=1, \ldots, N\right\}$.

$\mathrm{Wj}=\left(\mathrm{w}_{\mathrm{j} 1}, \ldots,\right)$ is the weights vector of each category $1 . . \mathrm{m}$

$\mathrm{I}_{\mathrm{i}}$ : is the input vector in the interval $[0,1]$.

$\|\mathrm{w}\|=\sum_{\mathrm{k}=1}^{\mathrm{n}}|\mathrm{w}|$ is the norm $\mathrm{w}$ vector

$\|\mathrm{I}\|=\sum_{\mathrm{k}=1}^{\mathrm{n}}|\mathrm{I}|$ is the norm of vector $\mathrm{I}$ 
The learning rule for updating the weight vector of the winning neuron can be defined by formula (7)

$$
\mathrm{W}_{\mathrm{ji}}(\text { new })=\mathrm{W}_{\mathrm{ji}} \text { (old)- } \beta^{*} \mid \mathrm{I}_{\mathrm{i}}-\mathrm{W}_{\mathrm{ji}}(\text { old }) \mid
$$

If $W j i<0$ then set $W j i=0$.

In the proposed learning rule, weights of the winning category also include two terms. The first term is the old weight vector, and the second term is the reduced category weights. The decrease of category weights depend on the learning parameter, and the difference between the input and the old weight vector.

\section{METHODOLOGY}

Generally, the color image (24 or 32 bits images or images in "true color" format) represents a bitmap, where each pixel is a convolution of RGB components [16-18]. This study deals with RGB images since this format is "natural" for all image capturing devices including; scanners and cameras. In such cases, the value of each pixel in true color format represents a set of three bytes, where each of byte carries the information about the red, green and blue colors, producing a total of 24 bits per pixel in the source image [17]. It is noted that the higher-order bits (D7) contain the majority of the visually significant data. The other bit planes contribute to more subtle details in the image [18]. Separating a digital image into its bit planes is useful for analyzing the relative importance played by each bit in the image, a process that aids in determining the adequacy of the number of bits used to quantize each pixel as shown in. Fig2. 


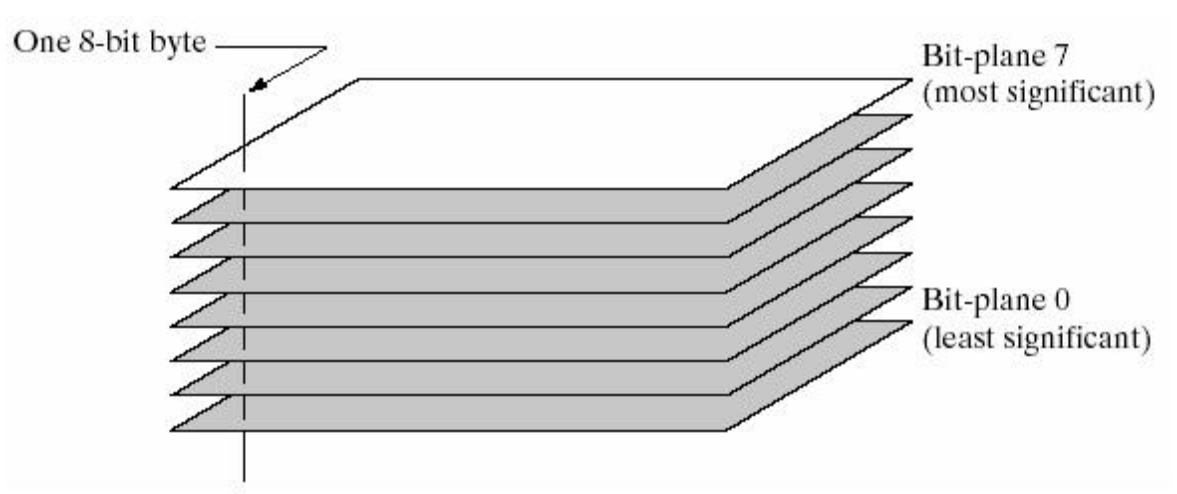

Fig. 2. The bit slicing

Any true color pixel is set of 3 numbers or R, G, B components. Each component is number in the range $\{0 \ldots 255\}$. For true color formats it is possible to use this method applying bit plane slicing for R, G, B components of pixel separately.

In contrast to highlighting gray-level ranges, highlighting the contribution made to the total image appearance by specific bits is desirable. Imagine that the image is composed of eight 1bit planes, ranging from bit-plane 0 for the least significant bit to bit-plane 7 for the most significant bit. In terms of 8-bit bytes, plane 0 contains all the lowest order bits in the bytes comprising the pixels in the image while bit plane 7 contains all the high-order bits as shown in Fig. 2. The other bit planes contribute to more subtle details in the image. Separating a digital image into its bit planes is useful for analyzing the relative importance played by each bit of the image, a process that aids in determining the adequacy of the number of bits used to quantize each pixel. Moreover, this type of decomposition we can use for fuzzy ART true color image recognition.

In [19], it was shown that in real images, most significant information was concentrated in first 4 (D7-D4) that allows the use 12-bit encoding without loss of the image visual quality. This study had also showed that in practical applications it is possible to use 6- bit encoding or 3 bit encoding. The color code range in each color encoding model could be assigned an integer value.

It is known, that fuzzy ART networks are intended for use with real value vectors. Thereafter, when applying fuzzy ART for color image recognition applications, images represented in 24bit RGB color encoding should be converted to vectors of real values using the bit slicing method and equation 8. In this work the source image represented in 24 bit color depth is transformed to images represented using lower color depth with a color encoding specification which assigns a digital code value of the location in the color space. Tables 1,2, 
and 3 shows the color encoding for 3-bit color image, a 6 bit color image and 12-bit color image respectively.

\section{Normalized color code $=$ color code $/$ max color code range}

Formula 8 results with real values ranging from [0..1], that means that pixel value is represented by real value. A software program was developed to acquire 24 bit source color face images and produce images with lower color depths by applying color encoding specification as shown in Fig. 3.

Table 1. 3-bit color encoding

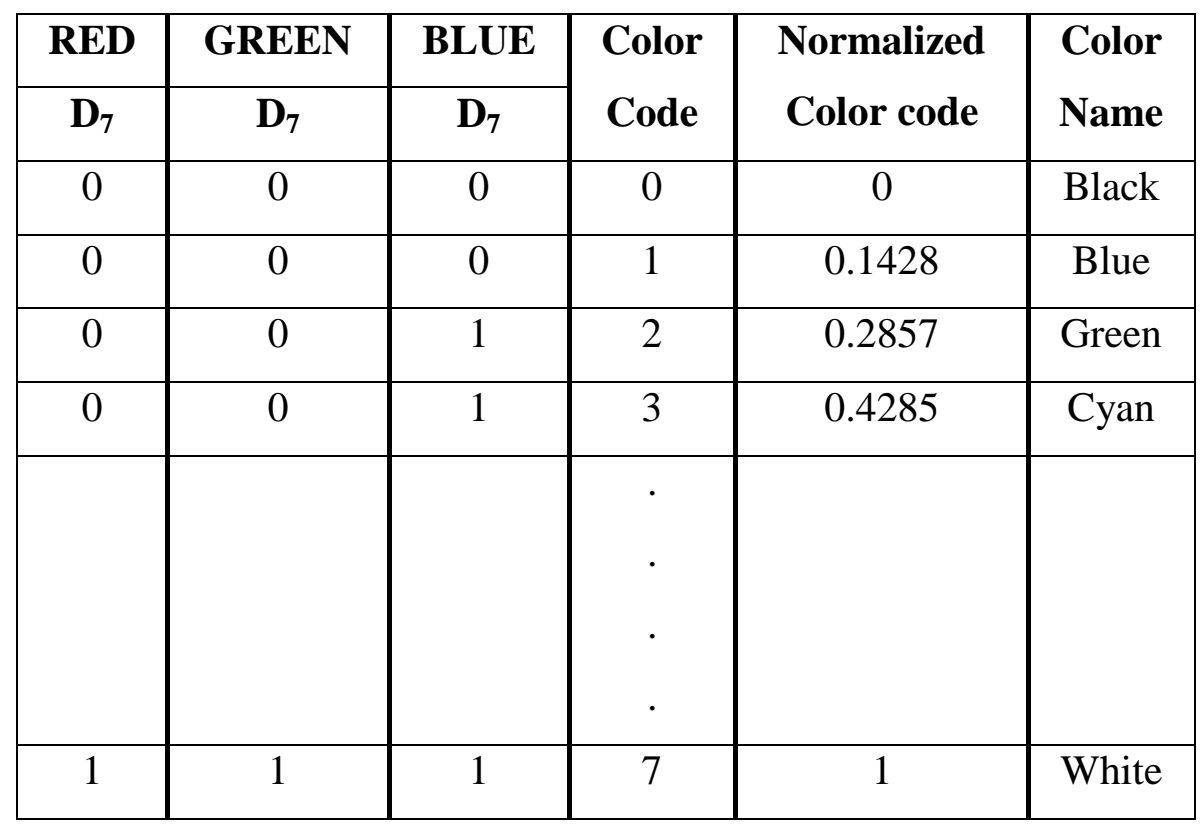


Table 2. 6-bit color encoding and normalized color code

\begin{tabular}{|c|c|c|c|c|c|c|c|}
\hline \multicolumn{2}{|c|}{ RED } & \multicolumn{2}{c|}{ GREEN } & \multicolumn{2}{c|}{ BLUE } & Color & Normalized \\
CD & $D_{\mathbf{6}}$ & $\mathbf{D}_{\mathbf{7}}$ & $\mathbf{D}_{\mathbf{6}}$ & $\mathbf{D}_{\mathbf{7}}$ & $\mathbf{D}_{\mathbf{6}}$ & Code & Color \\
\hline 0 & 0 & 0 & 0 & 0 & 0 & 0 & 0 \\
\hline 0 & 0 & 0 & 0 & 0 & 1 & 1 & 0.0158 \\
\hline 0 & 0 & 0 & 0 & 1 & 0 & 2 & 0.0317 \\
\hline 0 & 0 & 0 & 0 & 1 & 1 & 3 & 0.0476 \\
\hline & & & & & & $\cdot$ & \\
& & & & & & & $\cdot$ \\
\hline 1 & 1 & 1 & 1 & 1 & 1 & 63 & 1 \\
\hline
\end{tabular}

Table 3. 12-bit color encoding

\begin{tabular}{|c|c|c|c|c|c|c|c|c|c|c|c|c|c|}
\hline \multicolumn{7}{|c|}{ RED } & \multicolumn{5}{c|}{ GREEN } & \multicolumn{5}{c|}{ BLUE } & $\begin{array}{c}\text { Color } \\
\text { Code }\end{array}$ & $\begin{array}{c}\text { Normalized } \\
\text { Color }\end{array}$ \\
\hline $\mathbf{D}_{\mathbf{7}}$ & $\mathbf{D}_{\mathbf{6}}$ & $\mathbf{D}_{\mathbf{5}}$ & $\mathbf{D}_{\mathbf{4}}$ & $\mathbf{D}_{\mathbf{7}}$ & $\mathbf{D}_{\mathbf{6}}$ & $\mathbf{D}_{\mathbf{5}}$ & $\mathbf{D}_{\mathbf{4}}$ & $\mathbf{D}_{\mathbf{7}}$ & $\mathbf{D}_{\mathbf{6}}$ & $\mathbf{D}_{\mathbf{5}}$ & $\mathbf{D}_{\mathbf{4}}$ & & \\
\hline 0 & 0 & 0 & 0 & 0 & 0 & 0 & 0 & 0 & 0 & 0 & 0 & 0 & 0 \\
\hline 0 & 0 & 0 & 0 & 0 & 0 & 0 & 0 & 0 & 0 & 0 & 1 & 1 & $2.44 \mathrm{e}-4$ \\
\hline 0 & 0 & 0 & 0 & 0 & 0 & 0 & 0 & 0 & 0 & 1 & 0 & 2 & $4.88 \mathrm{e}-4$ \\
\hline 0 & 0 & 0 & 0 & 0 & 0 & 0 & 0 & 0 & 0 & 1 & 1 & 3 & $7.32 \mathrm{e}-4$ \\
\hline & & & & & & & & & & & & $\cdot$ & \\
& & & & & & & & & & & & $\cdot$ & \\
\hline 1 & 1 & 1 & 1 & 1 & 1 & 1 & 1 & 1 & 1 & 1 & 1 & 4095 & 1 \\
\hline
\end{tabular}




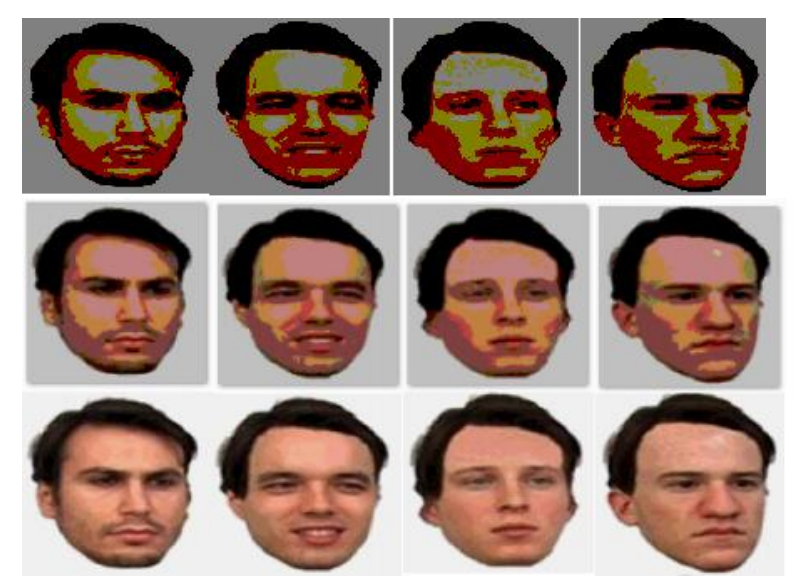

Fig. 3. Images represented in 3-bit, 6-bit and 12-bit color encoding

\section{EXPERIMENTAL RESULTS}

To study the practical results of enhanced recognition algorithms, software programs were developed in this research work using C\#. The modified fuzzy ART recognition algorithm was programmed in C\# and run on Intel(R) Core (TM) i5 CPU, $2.80 \mathrm{GHz}$, with input vectors of real values being extracted from RGB true color images (140x140 pixels). Images were selected from a public database of face images (Face images courtesy of the Face-Place Database Project, Michael J. Tarr ), using white backgrounds with different facial expressions as shown in Fig. 4

The developed recognition software consists of two parts:

A. Pre-processing of the true RGB color face images to obtain input vectors of real values, which are used as input to the modified fuzzy ART. The first person's facial images were first used as input to the system, followed by the second person's facial image. Thereafter, the remaining facial expressions of the other person samples were applied as images into the system. After the color face images were preprocessed according to mappings in tables 1 , table2, or table3, we obtained the vectors of real values. The results obtained were then used as inputs to the fuzzy ART neural network

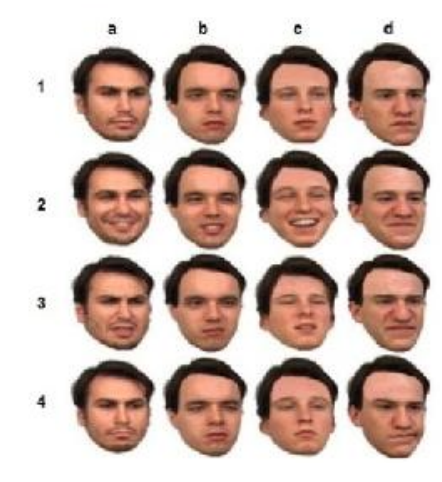

Fig. 4. Different color images of the person a, b,c,d with different facial expressions 
B. The enhanced fuzzy ART algorithm was applied on the resultant input vectors of real values. The input parameters of the modified fuzzy ART algorithm include the input vector of size 140x140 pixels, the vigilance parameter, and the learning rate.

The histogram of vectors extracted from the sample images is generated. The histogram is known as the codebook space information. The histogram stands by the feature vector of the human face. The histogram showed that the differences between the various facial expressions for one person is sometimes larger than the differences in expressions between two persons as shown in Fig. 5. During the learning and recognition process, the set of color face images (140x140 pixels) represented in normalized 12-bit color encoding is forwarded to the network input. The output of the network is the number of recognized clusters. We have provided two objectives in this study. The first objective estimates the ability of the network with vigilance parameter $\rho$ values $0.85,0.9,0.93$ and different learning rates $\beta$, to cluster the sequence of input face images for different persons. Recognition rate factors were used to estimate the ability of the network, and calculated using equation 9. Eighty color face images of twenty persons were input into network as a learning sequence of images of the first person, the second person, until the twentieth person. The results of the study is shown in table 4 . The second objective estimates the ability of the network with vigilance value $\rho=0.93$ and learning rate $\beta=0.055$ to recognize facial image sequences. The experimental results are shown in table 5.

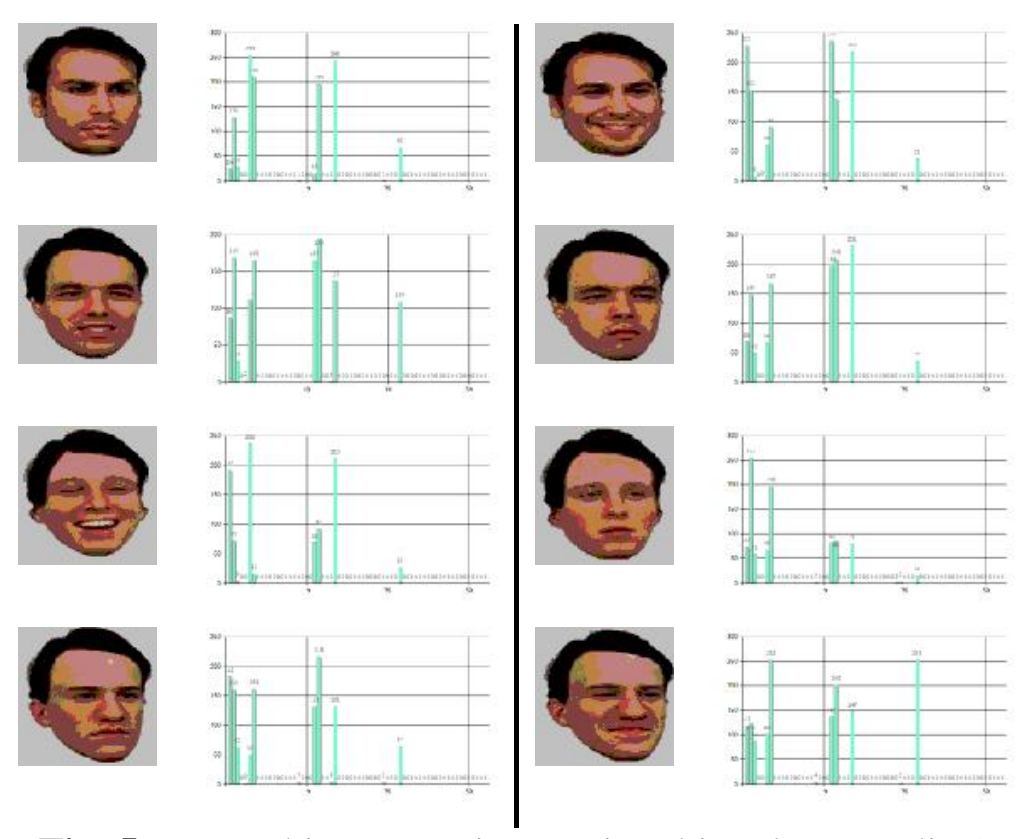

Fig. 5. Person histogram (images in 6 bit color encoding) 


$$
R r=\frac{\text { Number of true positives }}{\text { Number of truepositives }+ \text { Number of false Negatives }}
$$

Table 4. Clustering ability determined by recognition rate $\mathrm{Rr}$

\begin{tabular}{|c|c|c|c|c|c|}
\hline $\begin{array}{c}\text { Vigilance } \\
\rho\end{array}$ & $\beta$ & $\begin{array}{c}\text { Learning } \\
\text { images }\end{array}$ & $\begin{array}{c}\text { Number } \\
\text { of correct }\end{array}$ & $\begin{array}{c}\text { No. of } \\
\text { incorrect }\end{array}$ & $\mathrm{Rr}$ \\
\hline \multirow{3}{*}{0.85} & 0.1 & 80 & 67 & 13 & $83 \%$ \\
\cline { 2 - 6 } & 0.09 & 80 & 78 & 2 & $97 \%$ \\
\cline { 2 - 6 } & 0.085 & 80 & 78 & 2 & $97 \%$ \\
\hline \multirow{3}{*}{0.9} & 0.075 & 80 & 75 & 5 & $93 \%$ \\
\cline { 2 - 6 } & 0.07 & 80 & 77 & 3 & $96 \%$ \\
\cline { 2 - 6 } & 0.1 & 80 & 30 & 50 & $37 \%$ \\
\hline \multirow{3}{*}{0.93} & 0.05 & 80 & 75 & 5 & $93 \%$ \\
\cline { 2 - 6 } & 0.055 & 80 & 78 & 2 & $97 \%$ \\
\cline { 2 - 6 } & 0.056 & 80 & 77 & 3 & $96 \%$ \\
\hline
\end{tabular}


Table 5. Testing the recognition ability of the network (vigilance parameter $\rho=0.93$ and learning rate $\beta=0.055$ )

\begin{tabular}{|c|c|c|c|c|}
\hline $\begin{array}{c}\text { Test } \\
\text { number }\end{array}$ & $\begin{array}{l}\text { Learning } \\
\text { sequence }\end{array}$ & $\begin{array}{c}\text { Input } \\
\text { sequence }\end{array}$ & $\begin{array}{l}\text { The network } \\
\text { outputs } \\
\text { (number of } \\
\text { cluster) }\end{array}$ & Note \\
\hline 1. & $a, b, c, d$ & $\mathrm{a} 1, \mathrm{~b} 1, \mathrm{c} 1, \mathrm{~d} 1$ & $1,2,3,4$ & $\begin{array}{c}\text { All patterns } \\
\text { recognized correctly }\end{array}$ \\
\hline 2. & a,b,c,d & $\mathrm{b} 1, \mathrm{c} 1, \mathrm{~d} 1, \mathrm{a} 1$ & $2,3,4,1$ & $\begin{array}{c}\text { All patterns } \\
\text { recognized correctly }\end{array}$ \\
\hline 3. & $a, b, c, d$ & $\mathrm{c} 1, \mathrm{~d} 1, \mathrm{a} 1, \mathrm{~b} 1$ & $3,4,1,2$ & $\begin{array}{c}\text { All patterns } \\
\text { recognized correctly }\end{array}$ \\
\hline 4. & $a, b, c, d$ & $\mathrm{~d} 1, \mathrm{a} 1, \mathrm{~b} 1, \mathrm{c} 1$ & $4,1,2,3$ & $\begin{array}{l}\text { All patterns clustered } \\
\text { into two clusters (see } \\
\text { explanation below) }\end{array}$ \\
\hline 5 & $a, b, c, d$ & $\mathrm{a} 2, \mathrm{~b} 2, \mathrm{c} 2, \mathrm{~d} 2$ & $1,2,3,4$ & $\begin{array}{c}\text { All patterns } \\
\text { recognized correctly }\end{array}$ \\
\hline 6 & $a, b, c, d$ & $\mathrm{~b} 2, \mathrm{c} 2, \mathrm{~d} 2, \mathrm{a} 2$ & $2,3,4,1$ & $\begin{array}{c}\text { All patterns } \\
\text { recognized correctly }\end{array}$ \\
\hline 7 & $a, b, c, d$ & $\mathrm{c} 2, \mathrm{~d} 2, \mathrm{a} 2, \mathrm{~b} 2$ & $3,4,1,2$ & $\begin{array}{c}\text { All patterns } \\
\text { recognized correctly }\end{array}$ \\
\hline 8 & $a, b, c, d$ & $\mathrm{~d} 2, \mathrm{a} 2, \mathrm{~b} 2, \mathrm{c} 2$ & $4,1,2,3$ & $\begin{array}{c}\text { All patterns } \\
\text { recognized correctly }\end{array}$ \\
\hline
\end{tabular}

\section{CONCLUSION}

It was shown that it was possible to represent color images by using real vectors with color encoding specifications, which assign a digital code value to colors and normalizes the color code to obtain real values. The developed enhanced fuzzy ART algorithm was applied on RGB color face images for face recognition. Experimental results showed very good recognition rates were achieved when the value of vigilance parameter was in the range of $0.85-0.93$ with a learning rate of 0.05-0.1. It was noted that decreasing the value of the vigilance parameter had resulted with a decrease in the network accuracy. The work here had resulted with improved recognition rates for color images in comparison to previous related 
studies, as color cues played a role in face recognition, particularly when object shape cues were degraded.

\section{ACKNOWLEDGMENT}

This work has been carried out during sabbatical leave granted to the author Rashad Jamil Rasars from Al-Balqa' Applied University (BAU) during the academic year 2016/2017

\section{REFERENCES}

[1] G. Carpenter, S. Grossberg, and D. B. Rosen, "Fuzzy ART: Fast stable learning and categorization of analog patterns by an adaptive resonance system," Neural Networks, Vol. 4, 1991, pp. 759-771.

[2] MING GU, Fuzzy ART Neural Net and its Application, IEEE Xplore:28 Dec. 2009.

[3] Zhao W, Chellappa R, Rosenfeld A, Phillips P J. Face Recognition: A Literature Survey[J]. ACM Computing Surveys, 2003, 35(4):399-458.

[4] Wang Yao-Ming etc., “A Survey of Three-dimensional Face Recognition Research[J]”, Journal .

[5] H. Song, P. K. Hopke, M. Bruns, D. A. Bossio, and K. M. Scow, "A fuzzy adaptive resonance theory - supervised predictive mapping neural network applied to the classification of multivariate chemical data," Chemometrics and Intelligent Laboratory Systems. [6] M. Pacella, Q. Semeraro, and A. Anglani, "Adaptive resonance theory-based neural algorithms for manufacturing process quality control," International Journal of Pro- duction Research, Vol. 42, 2004, pp. 4581-4607.

[7] L.E. Parker, "Classification with missing data in a wireless sensor network," IEEE SoutheastCon, 2008, pp. 533-538

[8] N.C. Yeo, K.H. Lee, Y.V. Venkatesh, S.H. Ong. Color image segmentation using the self-organizing map and adaptive resonance theory // Image and Vision Computing, Volume 23, Issue 12, 1 November 2005, Pages 1060-1079.

[9] R. Kenaya and K. C. Cheok, "Euclidean ART neural networks," in Proceedings of the World Congress on Engineering and Computer Science, 2008, pp. 963-968.

[10] Nong Thiloa, An Improved Learning Rule for Fuzzy ART, Jouranl of Information Science and engineering 30, 713-726 (2014).

[11] Andrew W Yip, Pawan Sinhao, Contribution of color to face recognition, Perception, 2002, volume 31, pages $995-1003$. 
[12] H. Isawa, M. Tomita, H. Matsushita, and Y. Nishio, "Fuzzy adaptive resonance theory with group learning and its applications," in Proceedings of International Symposium on Nonlinear Theory and its Applications, 2007, pp. 292-295.

[13] H. Isawa, H. Matsushita, and Y. Nishio, "Improved fuzzy adaptive resonance theory combining overlapped category in consideration of connections," in Proceedings of IEEE Workshop on Nonlinear Circuit Networks, 2008, pp. 8-11.

[14] A. Yousuf and Y. L. Murphey, "A supervised fuzzy adaptive resonance theory with distributed weight update," in Proceedings of the 7th International Conference on Advances in Neural Networks, LNCS, No. 6063, 2010, pp. 430-435.

[15] G. Carpenter and M. Gjaja, Fuzzy-ART choice functions, Proc. World Congress on Neural Networks, I (1994), pp. 1-713 - 1-722.

[16] Clustering, in //Proceedings of the connectionist models summer school. Morgan Caufmann, LosAltros, CA, 1998, pp. 174-185.

[17] Gonzalez, R.C. and R.E. Woods, Digital Image Processing. 1992, Reading, Massachusetts: Addison-Wesley. 716.

[18] Castleman, K.R., Digital Image Processing. Second ed. 1996, Englewood Cliffs, New Jersey: Prentice-Hall.

[19] A new ART-1 Neural Network Interface Model Adopted for Color Images Recognition, Rashad J. Rasras; Ibrahiem M. M. EL Emary, D.E. Skopin, University of Pitesti - Electronics and Computers Science, Scientific Bulletin, No. 9, Vol. 1, 2009.

\section{How to cite this article:}

Jamil Rasras R. A fuzzy art neural network based color image Processing and recognition scheme. J. Fundam. Appl. Sci., 2016, 9(1S), 221-234. 